\title{
Assessment of Nutritional Services Provided for Hepatitis C Patients Attending the National Nutrition Institute in Cairo
}

\author{
YOSRA A. EL-SHAIKH, M.Sc.*; HANAN A. EL-RAGHY, M.D.**; HANAA A. ABOU-ZEINA, M.D. **; \\ DINA I. SHEHAB, M.D. ${ }^{* * *}$ and HANADY G. SHEHA, M.D.*** \\ The Department of Preventive Medicine, Faculty of Medicine, Helwan University*, \\ The Department of Public Health \& Community, Medicine, Faculty of Medicine, Cairo University** and \\ The Department of Clinical Nutrition, National Nutrition Institute***, Cairo
}

\begin{abstract}
Background: Malnutrition is common among chronic liver diseases patients. Thorough nutritional counseling should insure adequate dietary intake of different nutrients to prevent malnutrition and improves the prognosis. Maintenance of weight loss and exercise in overweight patients with liver disease results in a sustained improvement in liver enzymes, serum insulin levels, and quality of life. Adequate nutritional service is mandatory to overcome the challenge for obese Hepatitis $\mathrm{C}$ patients on a dietary regimen to supply adequate nutrients.
\end{abstract}

Aim of the Study: To assess the effect of nutritional counseling provided for obese Hepatitis $\mathrm{C}$ on their dietary pattern.

Subjects and Methods: This is an operational study that was conducted on NNI outpatient hepatic clinic. Two groups of those receiving and providing the nutritional services were interviewed; a sample of 120 obese Hepatitis C patients attending the NNI for at least the second time to follow-up their nutritional status (only 100 of patients agreed to complete the questionnaire and the 24 hour food intake recall with a response rate of $83.3 \%$ ), all the health care providers in the outpatient hepatic clinic were interviewed. Multiple data collecting tools were used; direct informal observation of activities for hepatic patients attending the NIOHC. Review of existing documents: Policies, protocols, standard operating procedures. Review of the health record system.

Results: The results of the analysis of the exit questionnaire showed insufficient provided services and insufficient satisfaction of studied patients.

Conclusion: This study concluded that there was lack of standard nutritional services provided for HCPs. Many factors have contributed to the ineffectiveness of this service. There are opportunities for improvement of the nutritional services provided to HCPs.

Key Words: Services assessment - Nutritional guidelines Nutritional services.

Correspondence to: Dr. Yosra A. El-Shaikh, The Department of Preventive Medicine, Faculty of Medicine, Helwan University

\section{Introduction}

EGYPT has the highest prevalence of the Hepatitis $\mathrm{C}$ Virus (HCV) in the world, with approximately 22 percent of Egyptian blood donors testing positive for the deadly disease. Lacking in infrastructure to deal with the virus, Egypt suffers from a particularly high morbidity and mortality rate, with 40,000 dying from the disease each year [1].

Different studies on chronic Hepatitis C patients concluded that even in the absence of cirrhosis patients with chronic viral hepatitis are prone to experiencing a decline in nutritional status $[2,3]$ Even in patients with well-compensated cirrhosis, malnutrition affected prognosis. Among those that were malnourished, $20 \%$ had a 1-year mortality rate, whereas none of the patients who received proper nutrition died within a 1-year period [4].

Intervention in the early stages of malnutrition in Hepatitis $\mathrm{C}$ patients can improve outcome [5] Lifestyle interventions including healthy eating, exercise and controlled weight loss have been shown to improve liver damage related to hepatic steatosis. Lifestyle changes should be an important adjuvant to medical therapies for patients with $\mathrm{HCV}$ infection, and NAFLD as well as other chronic hepatic conditions [6]

Maintenance of weight loss and exercise in overweight patients with liver disease results in a sustained improvement in liver enzymes, serum insulin levels, and quality of life [8]. The benefits of low-calorie diet and low-fat diet in management of patients with Hepatitis $\mathrm{C}$ regarding improvement of insulin resistance, steatosis and also fibrosis were shown in many studies [7]. 
It is important that gastroenterologists be proactive regarding nutritional counseling and both patients and their primary care physicians understand the importance of not imposing unnecessary restrictions on dietary intake [8] .

Early and evidence-based nutritional interventions are eagerly needed to minimize the nutritional decline associated with chronic liver disorders and ultimately improve the prognosis of such patients [9]. Future research should focus on identifying modifiable factors that affect quality of life of hepatic patients and can be targeted for improvement [10] .

In Egypt the National Nutrition Institute established since 1955 to provide the nutrition services for patients attending the outpatient clinics of the institute. It also provides nutrition education for Egyptian citizens through different media tools and educating booklets and handouts or through direct nutrition education sessions for patients attending the out clinics of the institute. The institute provides its services through general and specialized out clinics and other related units that works under the supervision of nutrition consultants and specialists. The institute provides nutritional services for patients with chronic diseases [11].

Due to an increasing number of hepatic patients in need of nutritional guidance and counseling, the outpatient clinics affiliated to the NNI, are probably facing difficulties with work pressure. Nonetheless, multiple evidences are needed to explore the unmet expectations of the attendant patients [12]

\section{Subjects and Methods}

Study site: National Nutrition Institute's Outpatient Hepatic Clinic in Cairo (NIOHC).

Study design: An interventional operational study. Situation analysis was done to identify the actual activities of nutritional services and its different components. Challenges and opportunities for improvement of the services were pointed, in order to identify areas to be addressed and plan for appropriate solutions. A policy brief was formed to be introduced to the NIOHC manager for opportunities of improvement.

Study subjects: The study included two groups of those receiving and providing the nutritional services in the NIOHC:

A purposive sample was taken from hepatitis $\mathrm{C}$ patients attending the NIOHC for following-up their nutritional status during the period of data collection (January 2015-December 2015). Where, out of a total of 120 patients; 100 agreed to complete the questionnaire, with a response rate $83.3 \%$. Patients who approved to participate were interviewed at the end station for their round in the NIOHC.

All health care providers actively working in the NIOHC at the time of the study were interviewed by the researcher. (The clinical nutrition physician, the dietitians and the nurse).

Study phases: This study was conducted on three phases:

I- Preparatory phase:Getting the formal agreement to conduct the study in the NNI (Annex 1).

- Reviewing literature and available standards for nutritional services for compensated hepatitis $\mathrm{c}$ patients in order to identify criteria and standards. Basically, it was needed to define the standards of nutritional services for them against which the services will be compared. So it was difficult to reach national standards of nutrition in compensated hepatitis c patients, except that found in the NIOHC. Unfortunately no available written standards were found there. So the Canadian Guidelines for Nutrition care of Hepatitis C (2003) $\&$ Guidelines on nutritional management in Japanese patients with liver cirrhosis, (2012) and ESPEN nutrition in liver disease, (2006) were revised for standards of nutritional care in compensated Hepatitis C patients.

- Preparing the study tools by reviewing different study methods and tools used for assessment of service delivery.

- Pilot study for the prepared exit interview questionnaire.

- Preparing administrative issues and ethical permission needed for conduction of work.

II- Data collection phase: For the assessment of the actual nutritional services provided in the NIIOHC and its effect on HCPs attending the NIOHC. five methods were used including:

A- Direct informal observation: To what is going on for hepatic patient attending the NIOHC. A flow chart for patients coming for the first time was done and another flow chart for those following-up in the NIOHC, to describe the following.

i- Work load: The available documents were revised for the number and clinical categories of the attendant Hepatitis $\mathrm{C}$ patients in the latest four year quarters. This is to calculate the average work load in the concerned clinics. 
ii- Service delivery: Service delivery assessment was organized into the following areas:

a- Adequate physical health systems facilities and equipment.

b- Executed activities and service delivery:

- Organization of Health Service: Existence and description of referral system and patients' flow charts and total turnaround time and waiting times.

- Access to health service: Physical and financial accessibility.

- Nutrition education: Nutrition education sessions pamphlets.

- Assessing whether service providers perform according to standard policies and procedures. Assessing whether these standards are adapted into a practical form that providers can use, such as clinical guidelines or manuals, job aids, charts, forms, checklists, or poster.

iii- Human Resources Management: Assessment profile included the following components:

- Current situation of health care providers: The physician, the nurse and the dietitian in terms of qualifications and duty rotation.

- Education and training activities: This process was investigated for pre-service education, inservice and specialty training including professional development. Frequency and quality of on job training.

iv- Record keeping system: Assessment of record keeping system was done by assessing the following areas:

a- Existence of policies or procedures for data entry, data access, data management, medical records storage, retrieval, maintenance and keeping. Also, policies or procedures for data security, review property or for quality control.

b- Infrastructure and physical resources for paper based medical records.

c- Data storage: Organization of records in such a way that they can be accessed easily while maintaining patient confidentiality. Records management system: Storage, retrieval and maintenance.

B- Review of existing documents: Policies, protocols, standard operating procedures and the record keeping system:

Medical record keeping and filing system whether computerized or paper based (Annex 4) were reviewed for the following: Sources of data, coding, completeness, utilization to obtain information to be used for decision making and planning.

C- Semi structured exit interview questionnaire with the attendant follow-up patients: The questionnaire was prepared in Arabic, with most of the questions closed ended. Themes of this questionnaire included:

- Their personal and socio-demographic data (name, sex, age, residency, education, marital status and work).

- Their medical history (their current height and weight measurement, duration of Hepatitis $\mathrm{C}$ infection, concomitant diseases, receiving antiviral treatment for $\mathrm{HCV}$ ).

- The services they received in the NIOHC, their attitude towards and adherence to these services, their acquired knowledge about nutrition in $\mathrm{HCV}$ infection.

- Their satisfaction; the questionnaire included direct and indirect questions about patients' satisfaction, the degree of benefit from their visit, recommending the institution for other patients, difficulties faced them, whether their expectations are fulfilled, and any raised suggestions to improve the delivered nutritional services.

D- 24 hour food recall intake: A full 24 hour food recall intake was done for the study patients.

III- Data processing and analysis:

Data obtained from direct observations were described in flowcharts.

Quantitative data from the questionnaire was coded and analyzed using (SPSS) program. Descriptive statistics were done.

Data obtained from 24 hour food recall analysis was analyzed according to food composition tables of the NNI [33], using SPSS 21 program. Descriptive statistics were done and analytical statistics were done to compare between subgroups using manwhitney test.

\section{Ethical considerations:}

- Verbal consent was obtained from all study participants.

- Privacy of participants as well as confidentiality of data collected were ensured by giving each participant a serial number.

- Collected data was used only for the stated research purpose. 


\section{Results}

This study assessed the nutritional services provided to Hepatitis $\mathrm{C}$ patients attending the NIOHC. The aim was to improve the nutritional services provided to these patients. Results are divided into two sections.

I- Results of the exit interview questionnaire of the studied patients.

II- Description and analysis of the activities delivered to hepatitis c patients attending the NIOHC.

\section{I- Results of the studied patients' exit interview questionnaire:}

In this study 100 Hepatitis $C$ patients attending the NIOHC for following-up were interviewed to assess the nutritional services they received, their knowledge about nutrition in Hepatitis C, practice of recommended therapeutic life style changes and attitude towards the nutritional services provided to them in the NIOHC.

Table (1) shows that the majority of the studied patients $(58 \%)$ were females. About two thirds of them $(66 \%)$ were aging $40-<60$ years. Residents of rural areas were nearly equal to those of urban areas; $51 \%$ and $49 \%$ respectively. Most of the studied patients were married $(77 \%)$.

Table (1): Personal characteristics of the studied patients.

\begin{tabular}{lcc}
\hline & $(\mathrm{N} .=100)$ & $\%$ \\
\hline Sex: & & \\
$\quad$ Female & 58 & 58.00 \\
Male & 42 & 42.00 \\
Age (years): & & \\
18<40 & 9 & 9.00 \\
$40<60$ & 66 & 66.00 \\
>60 & 25 & 25.00 \\
Residence: & & \\
Rural & 51 & 51.00 \\
Urban & 49 & 49.00 \\
Marital state: & & \\
Married & & \\
Others (divorced/widows/never married) & 77 & 77.00 \\
Education: & 23 & 23.00 \\
Illiterate & & \\
Read and write & 45 & 45.0 \\
Basic education & 20 & 20.0 \\
Secondary & 34 & 34.00 \\
Occupation: & 1 & 1.00 \\
No formal job & & \\
Farmer & & \\
Technician & 55 & 55.00 \\
Employee & 22 & 22.00 \\
\hline & 21 & 21.00 \\
\hline & 2 & 2.00 \\
\hline
\end{tabular}

Studied patients were of low educational level as about half of them (45\%) were illiterate while the highest educational level was the secondary education and was achieved only by $(1 \%)$. About of the $(77 \%)$ of them were either with no formal work or farmers, about (61\%) of them had one or more other chronic concomitant diseases.

Table (2) shows that, $61 \%$ of the studied patients had one or more other chronic concomitant diseases.

Table (2): Medical history of the studied patients.

\begin{tabular}{lcc}
\hline \multicolumn{3}{c}{ Other concomitant chronic diseases* } \\
\hline No diseases & 39 & 39.00 \\
Diabetes & 35 & 35.00 \\
Gastrointestinal & 24 & 24.00 \\
Cardiovascular & 17 & 17.00 \\
\hline
\end{tabular}

Table (3) shows that almost all of the studied patients $(98 \%)$ were obese.

Table (3): The studied patients according to BMI.

\begin{tabular}{lcl}
\hline & $\mathrm{N} .=100$ & $\%$ \\
\hline Obese $\left(\mathrm{BMI}>30 \mathrm{~kg} / \mathrm{m}^{2}\right)$ & 98 & 98.00 \\
Overweight $\left(\mathrm{BMI}=25-29.9 \mathrm{~kg} / \mathrm{m}^{2}\right)$ & 0 & 0.00 \\
Normal weight $\left(\mathrm{BMI}>18.4-24.9 \mathrm{~kg} / \mathrm{m}^{2}\right)$ & 1 & 1.00 \\
Underweight & 1 & 1.00 \\
\hline
\end{tabular}

Table (4), shows that (72\%) of them were attending the NIOHC to lose weight for different reasons. The majority of the studied patients (62\%) used to follow-up every month. Studied patients adherent to the follow-up schedule were $53 \%$ while $47 \%$ were non-adherent.

Table (4): The studied patients' utilization pattern of the NIOHC.

\begin{tabular}{lcc}
\hline & N.=100 & $\%$ \\
\hline Number of follow-up visits ${ }^{*}:$ & & \\
2nd or 3 rd & 48 & 48.00 \\
4th to 5 th & 33 & 33.00 \\
6th or more & 19 & 19.00 \\
& & \\
Reasons patients attend the NIOHC: & 41 & 41.00 \\
To lose weight to get better & 31 & 31.00 \\
To lose weight to receive antivirals & 20 & 20.00 \\
Seek nutritional guidance & 8 & 8.00 \\
Others ** & & \\
Patien ts' adherence to the follow-up visits: & 53 & 53.00 \\
Yes & 57 & 47.00 \\
No & & \\
\hline
\end{tabular}

* : Patients with first visit were excluded.

**: Others (Ex. abnormal bowl habits and to gain weight).

Fig. (1) shows that the majority of the studied patients (59\%), were advised by the physician of the NIOHC to follow-up every month. 


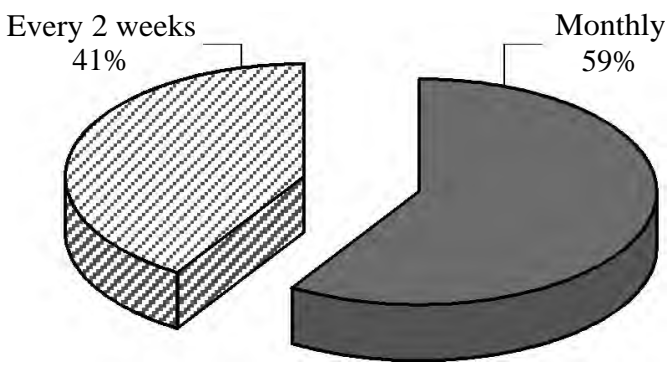

Fig. (1): The studied patients' frequency of attending the $\mathrm{NIOHC}$ as recommended by the physician.

Fig. (2) shows that most of the studied patients (78\%) were referred to the NIOHC from different medical facilities especially the liver institute $(41 \%)$.

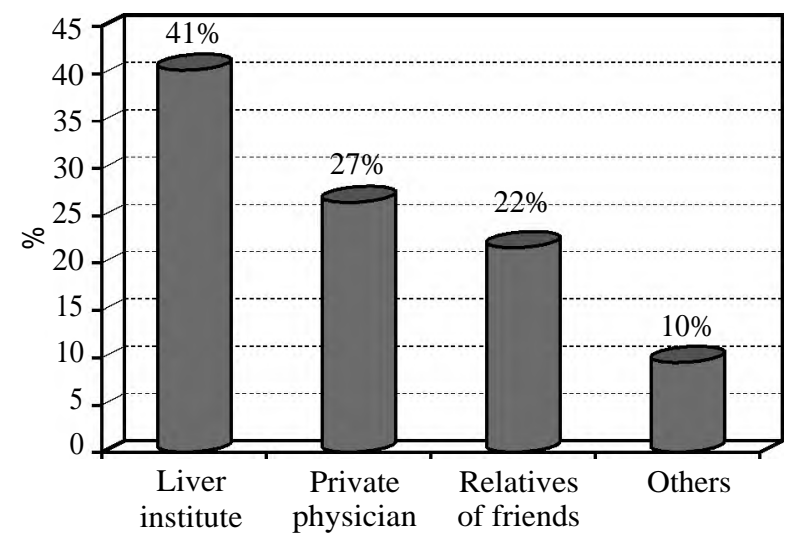

Fig. (2): The studied patients by the entity directed them to the NIOHC*.

*: Others (Ex. Districts and insurance hospitals).

Fig. (3) shows that (51\%) of the studied patients who weren't adherent to follow-up visits claimed that the journey to the NIOHC was costly and distant while about one third of them $(36 \%)$ accused that it is unaffordable service.

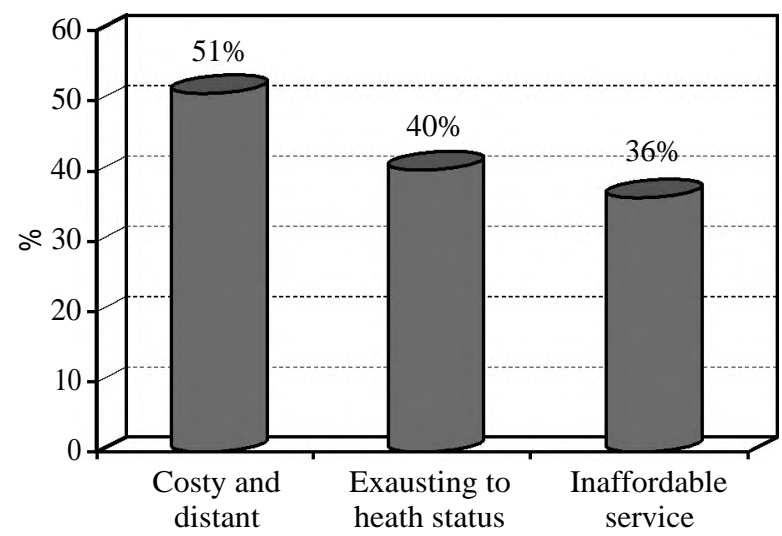

Fig. (3): Reasons the studied patients didn't adhere to the follow-up visits to the NIOHC*

\footnotetext{
*: - The studied patients that were not adherent to follow-up were only included, (47\%).

- Count adds to more than $100 \%$ due to multiple responses.
}

Table (5) shows that all the studied patients $(100 \%)$ had their weight measured. All of them confirmed that they didn't have their arm circumference measured. All the studied patients were not examined clinically. All of them didn't undergo lab investigations for nutritional assessment. All the studied patients received some nutritional education in the dietitian's room of the NIOHC. All of them didn't receive any nutritional education during waiting times. All of them stated that they didn't receive food or drug supplementations.

Table (5): Nutritional services the studied patients received in their current visit to the NIOHC.

\begin{tabular}{lll}
\hline & $\mathrm{N} .=100$ & $\%$ \\
\hline 1- Nutritional assessment: & 100 & 100.00 \\
$\quad$ Weight measurement & 0 & 0.00 \\
Arm Circumference (AC) & 0 & 0.00 \\
Clinical examination & 0 & 0.00 \\
Lab. investigation & & \\
2- Nutritional education: & 100 & 100.00 \\
Nutritional education in the dietitian room & 0 & 0.00 \\
Nutritional education during waiting time & 0 & \\
3- Nutritional supplementation: & & \\
$\quad$ Food or drug supplementations & 0 & 0.00 \\
\hline
\end{tabular}

Table (6) shows that two thirds of the studied patients $(66 \%)$ thought the nutritional services they received in the NIOHC were sufficient.

Table (6): The studied patients' opinions regarding adequacy of nutritional services they received in their current visit to the NIOHC.

\begin{tabular}{lcc}
\hline Opinion & N.=100 & $\%$ \\
\hline Sufficient & 66 & 66.00 \\
Not sufficient & 18 & 18.00 \\
Don't know & 16 & 16.00 \\
\hline
\end{tabular}

Table (7) shows that most of the studied patients $(81 \%)$ confirmed that they spent 2 or more hours in the NIOHC till exit.

Table (7): Time spent by the studied patients in their current visit to the NIOHC till exit.

\begin{tabular}{lcc}
\hline Time spent in the current visit & N. $=100$ & $\%$ \\
\hline$>3$ hours & 34 & 34.00 \\
$2-<3$ hours & 47 & 47.00 \\
$1-<2$ hour & 19 & 19.00 \\
\hline
\end{tabular}

Table (8) shows that $(50 \%)$ and $(24 \%)$ of the studied patients stated that they had much and very much benefits from following-up in the NIOHC respectively. 
Table (8): The studied patients' attitude towards attending the NIOHC.

\begin{tabular}{lcc}
\hline & $\mathrm{N} .=100$ & $\%$ \\
\hline Extent benefits from attending NIOHC: & & \\
$\quad$ Very much & 24 & 24.00 \\
Much & 50 & 50.00 \\
Average & 26 & 26.00 \\
Little & 0 & 0.00 \\
$\quad$ Very little & 0 & 0.00 \\
Recommending the NIOHC to other & & \\
Hepatitis C patients: & & \\
$\quad$ Yes & 52 & 52.00 \\
No & 48 & 48.00 \\
\hline
\end{tabular}

About two thirds of the studied patients (65\%) thought that the NIOHC is the best place for Hepatitis $\mathrm{C}$ patients to follow-up their nutritional status. The studied patients who recommended the NIOHC to other Hepatitis $\mathrm{C}$ patients were nearly equal to those who didn't recommend it (52\%) and (48\%) respectively.

Table (9) shows that (71\%) of the studied patients knew Hepatitis $\mathrm{C}$ has effect on the nutritional status. About half of the studied patients (53\%) thought that the balanced diet is the best for hepatitis c, while (42\%) of them knew they should have more frequent fish meals. Most of them (88\%) believed that over or under weight affects Hepatitis C.

Table (9): Knowledge of the studied patients about nutrition in Hepatitis $\mathrm{C}$ infection.

\begin{tabular}{lcc}
\hline & N.=100 & $\%$ \\
\hline $\begin{array}{l}\text { Hepatitis C infection has negative effect } \\
\text { on the nutritional status: }\end{array}$ & & \\
$\quad$ Yes & 29 & 29.00 \\
No & & \\
Best diet for Hepatitis C patients *: & 53 & 53.00 \\
$\quad$ Balanced with all nutrients & 42 & 42.00 \\
$\quad$ More frequent Fish & 26 & 26.00 \\
$\quad$ Low in meat and fat & & \\
Negative effect of increased or decreased & & \\
weight on Hepatitis C infection: & 88 & 88.00 \\
$\quad$ Yes & 12 & 12.00 \\
$\quad$ No & & \\
*: Count adds to more than 100\% due to multiple responses. &
\end{tabular}

Table (10) shows that, two thirds of the studied patients $(66 \%)$ were adherent to therapeutic lifestyle changes prescribed by the dietitian.

Table (10): Adherence of the studied patients to therapeutic lifestyle changes prescribed for them by the dietitian of the NIOHC.

\begin{tabular}{lcc}
\hline $\begin{array}{l}\text { Adherence to the therapeutic } \\
\text { lifestyle changes }\end{array}$ & N.=100 & $\%$ \\
\hline Yes & 66 & 66.00 \\
No & 34 & 34.00 \\
\hline
\end{tabular}

Table (11) shows that about half of the nonadherent studied patients $(49.8 \%)$ to the therapeutic lifestyle changes did not adhere because they couldn't prepare healthy food, while about one third of them (34.5\%) did not adhere because they couldn't perform the physical activity component prescribed for them.

Table (11): The studied patients' reasons of non-adherence to the therapeutic lifestyle changes.

\begin{tabular}{lcc}
\hline Reasons of non-adherence* & N. $=34^{*}$ & $\%$ \\
\hline Can't prepare healthy food & 17 & 49.8 \\
Can't do the physical activity component & 12 & 34.5 \\
Out home all the time & 10 & 29.4 \\
Expensive food & 5 & 14.5 \\
\hline
\end{tabular}

*: - The only $34 \%$ of the studied patients who didn't adhere to diet regimens were included.

- Count adds to more than 100 due to multiple responses.

Table (12) shows that $69 \%$ of the studied patients were either satisfied or very satisfied from the nutritional services in the NIOHC.

Table (12): The studied patients' satisfaction about the nutritional services in the NIOHC.

\begin{tabular}{lcc}
\hline & $\mathrm{N} .=100$ & $\%$ \\
\hline Very satisfied & 11 & 11.00 \\
Satisfied & 58 & 58.00 \\
Neutral & 30 & 30.00 \\
Unsatisfied & 1 & 1.00 \\
Very unsatisfied & 0 & 0.00 \\
\hline
\end{tabular}

Table (13) shows that most of the studied patients $(89 \%)$ stated that long waiting time was the main difficulty faced hepatitis c patients attending the NIOHC.

Table (13): The studied patients' opinions about difficulties that may face Hepatitis $\mathrm{C}$ patients attending the NIOHC.

\begin{tabular}{lcc}
\hline & $\mathrm{N} .=100$ & $\%$ \\
\hline Long waiting time & 89 & 89.00 \\
Can't read written diet plan & 20 & 20.00 \\
Inapplicable diet plan & 7 & 7.00 \\
\hline
\end{tabular}

*: Count adds to more than $100 \%$ due to multiple responses.

Fig. (4) shows that all the studied patients $(100 \%)$ suggested decreasing waiting times. About third of the studied patients suggested followingup every two weeks and only $12 \%$ of them suggested following-up every week.

\section{II- Analysis of the activities delivered to hepatitis c patients attending the NIOHC:}

1- Setting: The NIOHC is located in the ground floor of the national nutrition institute with other out-patient complex, to the right of the main gate 
of it. No guiding signs were present either along the way to or on the clinic to help its easy identification.

2- Executed activities: The NIOHC served the Hepatitis C patients HCP (new and follow-up patients) only on Tuesdays from 9:00am to $2.00 \mathrm{pm}$ except on official vacations.

Fig. (5) demonstrates the flow cycle of the patients coming for follow-up visits.

Hepatitis C patients attending the NIOHC for follow-up had to buy a ticket from the tickets' office, which took about 5-15 minutes according to flow rate that day.

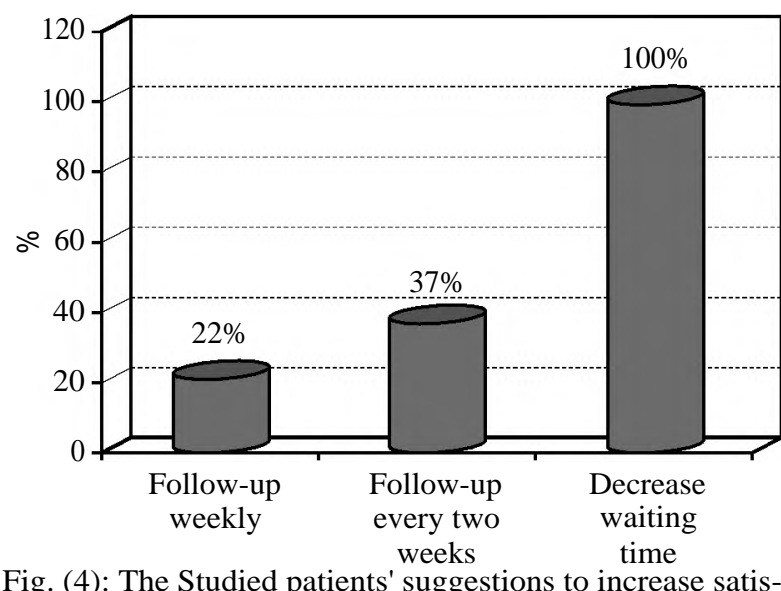

Fig. (4): The Studied patients' suggestions to increase satisfaction of Hepatitis $\mathrm{C}$ patients attending the NIOHC.

The flow cycle of Hepatitis C patients attending the NIOHC for the first time, November 2014

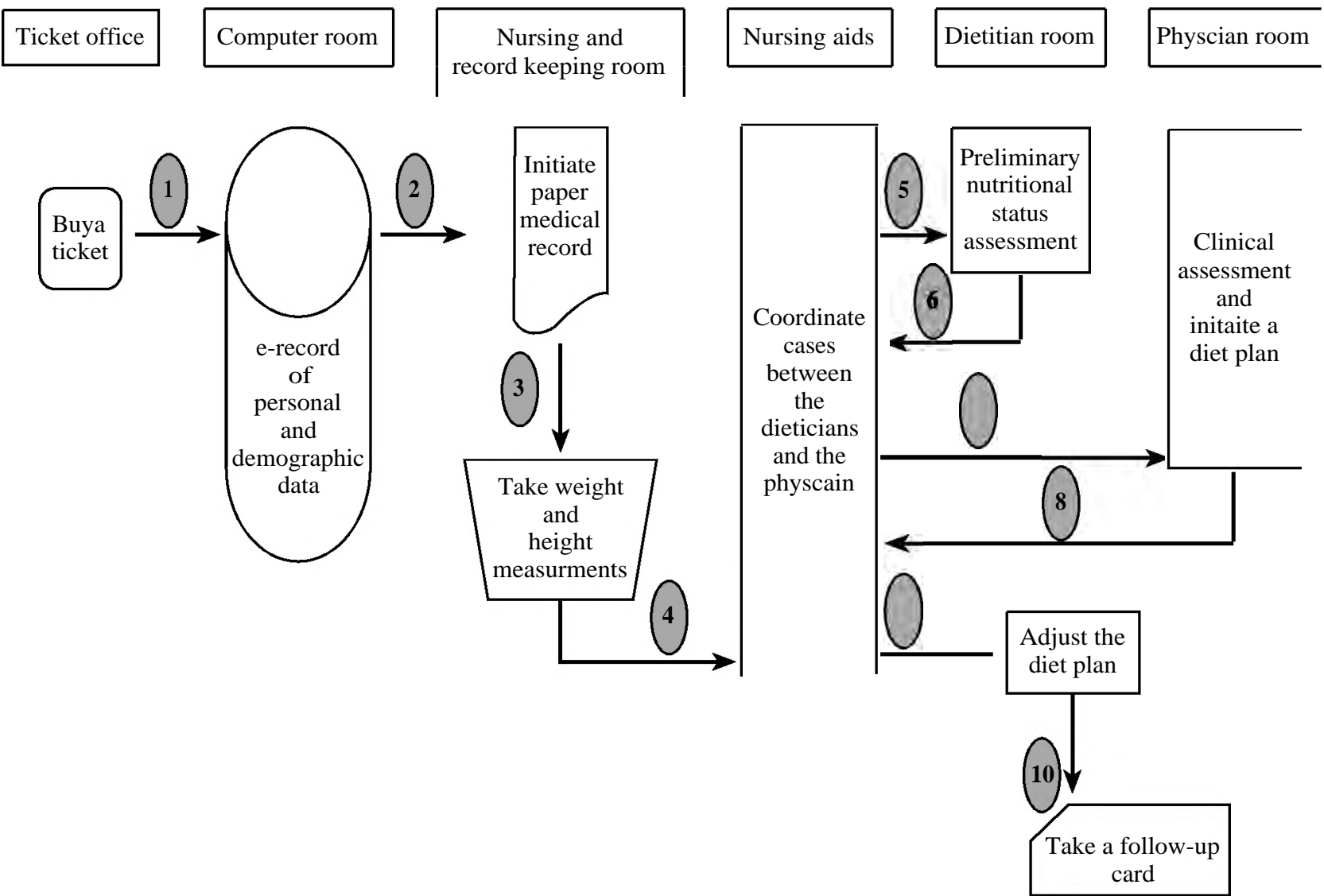

Fig. (5): The flow cycle of Hepatitis C patients attending the NIOH for the first time.

Then they were asked to wait in the backyard of the outpatient complex for 10-15 minutes till being called by the nursing aid to retrieve their paper medical records.

They went to the nursing and medical records keeping room where they had their paper medical records retrieved and had their body weight measured and recorded. This took 10-15 minutes.
They were asked to wait in the backyard of the outpatient complex for another 10-15 minutes till being called to encounter the dietitian.

Then they were called by the nursing aid to encounter the dietitian who assessed their adherence to the diet plan and factors of non adherence, made further nutritional assessment and change the prescribed food if needed. This took 10-15 minutes. 
They waited again in the backyard of the outpatient complex for 10-40 minutes, according to the flow rate, till being called by the nursing aid to encounter the physician of clinical nutrition.

They encounter the physician for medical recommendations and new diet plan for about 10-15 minutes.

They wait again in the backyard of the outpatient complex for 10-15 minutes till being called by the nursing aid to encounter the dietitian.

Then they were called by the nursing aid to return back to the dietitian to modify the prescribed meals and types of food in a written pamphlet that was given to them, in addition to any necessary nutritional guidance and advice. Some of them were given educational written brochures about low cholesterol diet and fasting. This took 10-15 minutes.

The paper medical records were retained in the clinical nutrition room to be collected at the end of the day and returned to their places in the cabinets by the nurse aid and worker. Some paper medical records were retained in the dietitian room.

The average time for the flow cycle of a following-up patient from entering the NIOHC till finishing his visit was about 122 minutes (about two hours). Waiting time was about 62.5 minutes in average.

3- Human resources: The NNI is served by a good number of clinical nutrition physicians and dietitians. However, the NIOHC is served by one clinical nutrition physician a dietitian and a nurse each time.

\section{1- Work load:}

The total number of medical paper records for all patients attending the NNI outpatient clinics found to be 11453 records in 2015 . The number of medical records of hepatic patients was (876 record) of which (563) were Hepatitis C patients with a percent of $64 \%$ of hepatic patients and $5 \%$ of all patients attending the NNI.

The number of medical paper records for HCPs attended for follow-up care in the same year was 321 records (57\% of HCPs), of which (226 records) for the first half of the year and only (94 records) in the second half of the year.

\section{2- Record keeping system:}

Computerized registration was done for basic data of patients with a serial number.
The paper based medical records of the patients were kept in lockers in the nursing and record keeping room.

\section{Paper medical record components:}

It is a medical paper record for all cases attending the outpatient clinics of the NNI and was not specific for hepatic cases. It was composed of 8 papers fulfilling about all the items.

\section{First page included:}

Basic data: (Visit date, name, ID, gender, birth date, residency and phone number).

Anthropometry: (Height, weight, BMI, waist, hip and waist/hip ratio and other specific measures for specific cases and children.

Clinical and nutritional assessment sheets: Contain three criteria of assessment (lifestyle and nutritional status-laboratory-clinical) which are often not completed for hepatic cases.

Follow-up sheet: Contains follow-up visits' data (weight, height and recommendations).

Food frequency and 24 hour food recall intake sheet: This is the last page which is sometimes lost or torn from the patient's paper medical record.

The researcher revised the completeness of the paper medical records of all the studied patients, findings are presented in Table (14).

The overall incompleteness of data were realized all through the different components of the revised paper records.

\section{3- Control measures of activities:}

- No written or distributed guidelines and procedures were available in the NIOHC.

- The NNI regulates an accredited diploma in clinical nutrition for physicians, dietitians, nurses who should be working in the institute. These courses adopt mainly ESPEN guidelines.

- The library of the NNI contains a lot of up to date books and references in nutrition in chronic liver diseases which are available for health care workers.

\section{4- Monitoring and evaluation of effectiveness of activities:}

The NNI is accredited by the ISO accreditation certificate which is renewable every 6 months.

The available data in the NIOHC were not used in the formulation of any clinical, financial, or 
operational indicators that reflect the performance of activities.

Table (14): Completeness of data recording in the paper medical records of the studied patients as revised by the researcher.

\begin{tabular}{lll}
\hline & $\mathrm{N}$ & $\%$ \\
\hline Personal data: & 100 & 100.00 \\
Name & 67 & 67.00 \\
Residency & 30 & 30.00 \\
Phone number & 0 & 0.00 \\
Work & 0 & 0.00 \\
Education & & \\
Medical history data: & 87 & 87.00 \\
Date of visits & 91 & 91.00 \\
Diagnosis as hepatic patient & 0 & 0.00 \\
Other concomitant diseases & & \\
Assessment items: & & \\
1- Clinical assessment: & 0 & 0.00 \\
Stage of liver disease & 0 & 0.00 \\
Severity of damage & 0 & 0.00 \\
Blood pressure & & \\
2- Anthropometric measurements: & 100 & $100 \%$ \\
Weight & 100 & 100.0 \\
Height & 0 & 0.00 \\
BMI* & 0 & 0.00 \\
MAC & 0 & 0.00 \\
WC & 0 & 0.00 \\
TSF & 0 & 0.00 \\
AMC & 0 & 0.00 \\
3- Results of lab. investigations** & 0.00 \\
*: BMI was recorded only by the clinical nutrition physician in the \\
patients follow-up sheets. \\
**: No results of any laboratory investigations & were recorded. \\
& &
\end{tabular}

\section{Discussion}

Egypt has the highest prevalence of the Hepatitis $\mathrm{C}$ Virus (HCV) in the world, with approximately 22 percent of Egyptian blood donors testing positive for the deadly disease. Lacking in infrastructure to deal with the virus, Egypt suffers from a particularly high morbidity and mortality rate, with 40,000 dying from the disease each year [1]

The National Nutrition Institute of Egypt (NNI) has been established since 1955. Through its outpatient clinics, it provides growing nutritional services to a wide verity of attendants including HCPs [11].

A "process" can be defined as a "set of interrelated or interacting activities, which transforms inputs into outputs". The process approach is a review of the sequence and interaction of processes and their inputs and outputs. The purpose of the process approach is to enhance an organization's effectiveness and efficiency in achieving its defined objectives [13].
This study adopted the process analysis in an attempt to pin point opportunities to improve the effectiveness and efficiency of the nutritional services provided for HCPs attending NIOHC [13] This study presented how process analysis is one of the systematic tools that would help NIHOC become more 'oriented' about how smooth, transparent, consistent are the operations within the NIHOC, as well, to adopt a more structured and rigorous-yet simplified-approach to problem solving.

In addition, the graphic display of the data using multi-dimensions flowchart used in this study enhanced understanding and the use of the results. This simplified the main processes in the NIHOC, and constituted an aid to organizational learning.

The maintenance of complete and accurate medical records is a requirement of health care providers. The medical record serves as the central repository for planning patient care and documenting communication among patient and health care provider and professionals contributing to the patient's care [14]

Unfortunately, the grave incomplete data recording in the paper medical records of the studied HCPs as revised by the researcher. Such incomplete information in the paper medical records, especially for the studied patients who almost all of them had other co-morbidities, would seriously hinder evaluating the effectiveness of the provided nutritional services.

This study highlighted the perception of the studied HCPs about the quality of the provided nutritional services at the NIHOC. More than two thirds were either satisfied or very satisfied.

In addition, two thirds of the study HCPs thought that the provided nutritional services provided for them in their current visit to the NIOHC were sufficient.

Because it is not merely necessary to be aware of how the service is perceived, but also how it is quantifiably delivered, the inclusion of the quantitative information about the delivered services would provide policy makers with an additional pointer to specific opportunities of improvement related to nutritional services' components provided for the attendant HCPs [15].

Strikingly, pointed at the limited span of nutritional services provided to the studied patients received in their current visit to the NIOHC. Though expectations impose important influence on the 
patient's overall satisfaction with a health care experience, the link between technical quality and fulfillment of patient expectations should not be always directly correlated. This is because it is possible that patient's satisfaction from a service may be largely independent of its actual technical quality. Observed differences in satisfaction between people from different social classes, age, sex and cultural group or between different services and types of care may be confounded by match or mismatch between expectation and the service received [16,17].

So, the studied HCPs' low level of expectations of adequate nutritional services were anticipated from such attendants with border line social levels as reflected from their overall modest social and educational levels.

The disease progresses slowly, subtle nutritional deficits are not always obvious at early stages. Therefore, it is vitally important that patients infected with HCV try to maintain a balanced diet with adequate energy, protein, carbohydrate, fat, vitamins, minerals and fluids. Eating well can become more of a challenge as liver disease progresses [18].

This study showed that although the studied HCPs were mostly aware about the negative effect of Hepatitis $\mathrm{C}$ infection on the nutritional status, value of early follow up of nutrition, their 24 hours food recall intake analysis reflected values that are far beyond the recommended dietary allowance.

This may be due to their poor precise knowledge about the components of the balanced diet they should consume. Table (9) shows that only about half of them were knowledgeable about a balanced diet with all nutrients as the best for the HCPs. Additional explanations were their fears from the influence of excessive weight gain on Hepatitis $C$ infection and/or their urged desire to lose weight (Table 4). The Dietitians of Canada, 2003, emphasized the impressive role of nutritional education and counseling in managing HCPs, this raises questions about the quantity and quality efforts of NIHOC regarding this perspective [18].

In addition (Table 5) pointed no nutrition education during waiting time. The dietary advice for HCPs, should be accurate, specific and sufficiently detailed to empower the patient to act on it [18].

The ultimate aim of this study was to have the nutritional services provided to the HCPs measured. As, what can't be measured, can't be improved.
Measuring the efficiency and effectiveness of a service would guide improvement initiatives and hot areas to deal with in the change process $[19,20]$.

Activity analysis of the nutritional services provided by the NIHOC, revealed that the available data in the NIHOC were not used in the formulation of any clinical, financial, or operational indicators that reflect the performance of activities. This is in addition to unavailable written or distributed standards, procedures or guidelines.

The starting point towards improved NIHOC nutritional services is inspiring transparent leadership that is sensitive to the principles of change management, sharing common vision and goals with the working team, and after all with unwavering commitment. Only at this point, initiatives for improvement can come true $[\mathbf{1 9 , 2 0 ]}$

Results of the exit interview questionnaire with the studied HCPs, revealed that about four fifth of them spent at least two hours and more in their current visit to NIOHC till exit, (Tables 7,13) shows that this long waiting time was the main difficulty faced by most of the studied HCPs. Fig. (4) demonstrates that the prime suggestion elaborated by all the studied HCPs was decreasing waiting time. Activity analysis revealed that this waiting time was spent on interrupted bases in moving among the nursing room, physician of clinical nutrition's room and the dietitian's room.

The interrupted nature of this long waiting time, make it difficult to provide intact health education messages to the attendant HCPs. The activity analysis presented in Fig. (5) ascertained also that long time passed in vain in moving among different health care providers. It pointed that the little number of the nursing aids and the dietitian is a contributing factor in prolonged waiting in the corridors of the NIHOC or its backyards. It pointed also that lack of any comprehensive map of the NIHOC or any direction signs contributed in missing the correct ways to the different rooms of the clinic and imposed an additional burden to the little number of the working nursing aids.

Lack of prompt addressing of the problem of long waiting time in governmental health facilities was partly explained that working in governmental health facilities without direct economic profit, may make some of the health care providers as well as the managerial board careless about understanding and consequently addressing what is critical to satisfaction of the attending patients, as long waiting time $[21,22]$ 
This finding may also implicitly reflect that the shortage by which the set policies and regulations of the governance of NIHOC regarding the patient's rights in receiving quality care has been communicated to the front line care providers [23].

In addition, the poor commitment of the NIHOC's physician of clinical nutrition to start work on time caused the launch of the perplexity in the patient flow. This observation supported the findings of many studies that emphasized that the poor supervision of health care personnel is a fairly common cited cause for inefficient health care delivery. Setting up a health program without provision of close and periodic supervision which is based on explicit criteria, most likely would result in wasted resources, lowered morale and work commitment [23-25]

This study revealed an expected conflict facing the studied HCPs. Most of them suggested followup at shorter time intervals. They thought that this would help them be more adherent to the prescribed medical instructions. Thus, they agree with other studies that rapport which is a positive or close relationship between people that often involves mutual trust, understanding and attention is built over time. The long, often irregular time intervals between the follow up visits, breaks this rapport to be established, and subsequently has its influence on compliance with the medical instructions and continuity of care $[\mathbf{2 1 , 2 3 ]}$

On the other hand, (Table 4) shows that, only about half of the studied HCPs were adherent to the follow-up visits. Un-modifiable factors seem to influence their wish to attend at closer time intervals. Inadequate number of dietitians as revealed from the in-depth interview and/or unavailable phone numbers to more than two thirds of the patients, make it very difficult in using phones as a means to communication and follow-up.

Risks of Steatosis occurs in more than $50 \%$ of overweight patients with chronic Hepatitis C. In many of these patients, the pathogenesis appears to be related to obesity [36-39]. Hepatic steatosis, especially when associated with obesity, is an important precursor to the development of fibrosis in Hepatitis $\mathrm{C}$ and other liver diseases [36,40] Weight reduction may provide an important adjunct treatment strategy for patients with chronic Hepatitis C. In overweight patients with steatosis who subsequently lose weight, liver-related abnormalities improve [27]

Although sustained weight loss may be difficult to achieve, weight loss in these patients may be associated with a reduction in steatosis and abnormal liver enzymes and an improvement in fibrosis, despite the persistence of the virus $[26,27,31]$

Weight loss has also been shown to decrease visceral fat. So, with the limited nutritional services received by the studied HCPs this study revealed that a big deal of studied HCPs-whom almost all are obese with only one half were adherent to the follow-up visits would suffer from the combined burden of obesity and HCV infection.

Dealing with issues related to the therapeutic lifestyle changes of the study HCPs including adequate nutrition as well as physical activity is always a challange. Dietitians of Canada ascertained that HCPs should be encouraged to obtain optimal nutritional status through a balance of food intake, physical activity and medication (if required) to enhance their immune system and promote liver regeneration [18]

This study found that only two thirds of the study HCPs claimed to be adherent to the therapeutic lifestyle changes prescribed by the dietitian.

Complex interacting factors were found to influence this recommended adherence to the therapeutic lifestyle changes. Nonetheless, (Table 9) shows that a big deal of the study HCPs were knowledgeable about the negative effect of Hepatitis $\mathrm{C}$ infection on the nutritional status, the value of early nutritional follow-up on being infected with $\mathrm{HCV}$, as well as the negative effect of increased or decreased weight on Hepatitis $\mathrm{C}$ infection. It is recommended that physicians and dietitians at the NIOHC should build on this promising finding and try harder the use of the most simple and attractive well designed illustrated leaflets, flyers or boosters to improve patients' adherence to sound lifestyle changes that best fit the style of the attendant patients, mostly with no or very limited education. Table (13) reflects the hardship encountered by about one quarter of the study patients in using the instructions.

The patients' profile in Egypt has changed. The number of patients who are elderly, chronic, polymedicated and living alone is on the rise [32]. This trend requires that those professionals involved in their care collaborate in order to optimize the results of the management and thus improve their patients' health. This paradigm shift in healthcare demands more personalized care and a comprehensive response to health problems through collaboration that ensures the patients' needs are constantly met. 
In this study it was found that the majority of patients were females of the age group 40-60. Most of them were of low socioeconomic level; about half of them were unemployed and illiterate. Almost all of them were obese and most of them had other chronic concomitant disease specially diabetes.

This study revealed the bad sequence of the absence of collaboration between different health care providers involved in managing HCPs, which discouraged HCPs attending other medical organizations to seek the proper nutritional care in the NIOHC regardless the need to lose weight to receive antiviral treatment. This was obvious from the dramatic decrease in HCPs' flow after the introduction of the new drug for HCV infection in the NHI.

\section{Conclusions:}

1- The provided nutritional services for the HCPs were not matching the adopted and other reviewed international guidelines regarding assessment, supplementations and nutrition education.

2- The nutritional services provided for HCPs in the NIOHC were not effective. That was revealed from the dietary profile of the studied patients which showed insufficient practice of the therapeutic life style changes mentioned in the guidelines.

3- There was lack of collaboration between NNI and other curative care organizations serving Hepatitis C patients specially NHI, although it's evident that improving nutritional status will prevent complications and improve the outcome of the disease. This discouraged patients to seek the proper nutritional care in the NIOHC.

4- Complex factors (doctor patient contact, distant follow up visits and insufficient nutrition education) imposed the patients' compliance to the therapeutic lifestyle changes.

5- Long total turnaround time with many long waiting time stations declined patients' satisfaction.

\section{Recommendations:}

1-Establishing a protocol for collaboration between NIOHC and NHI regarding management of HCPs will improve HCPs' flow and adherence to follow-up visits.

2- Adopting and disseminating the evidence based nutritional guidelines for managing HCPs in the NIOHC and ensure its implementation.
3- Assuring the implementation of the nutritional services provided according to guidelines.

4- Disseminating well designed illustrated brochures and lifestyle changing plans will improve patients' attention, recall, understand and adherence to therapeutic lifestyle changes.

5- Monitoring of the completeness of patients' data in their records can help in contacting patients by phone to encourage patients' adherence to follow-up visits.

6- More patient doctor contact by shortening the intervals of follow-up visits and contacting patients by phone between visits can improve patients' practice.

7- Monitoring and evaluation of the service delivery according to the guidelines.

\section{References}

1- MAMOUD Y.A., GHINA R.M., RIOME S., ABURADDAD J., et al.: The epidemiology of Hepatitis C virus in Egypt: A systematic review and data synthesis. BMC Infectious Diseases, 13: 288, 2013.

2- LIN C., LIU H., WANG H. and YIN C.: Vitamins B status and antioxidative defense in patients with chronic hepatitis B or Hepatitis C virus infection. Eur. J. Nutr., 50: 499506, 2011.

3- ISMAIL W., KHAN A., KAMANI L.,WADALAWALA A., et al.: Nutritional status in patients with Hepatitis C. J. Coll Physicians Surg. Pak., 22: 139-42, 2012.

4- ALVARES-DA-SILVA M. and SILVEIRA T.: Hand-grip strength or muscle mass in cirrhotic patients: Who is the best?. Nutrition, 22 (2): 218-219. Retrieved 27 Mar. 2016, from http://dx.doi.org/10.1016/j.nut.2005.06.001, 2006.

5- HENKEL A. and BUCHMAN A.: Nutritional support in patients with chronic liver disease. Nature Clinical Practice Gastroenterology \& Hepatology, 3 (4): pp. 202-9, 2006.

6- NOBILI V., CARTER-KENT C. and FELDSTEIN A.: The role of lifestyle changes in the management of chronic liver disease. B.M.C., 9-70, 2011.

7- RUSU E., RALUCA P. and RATZIU V.: The Impact of Obesity and Metabolic Syndrome on Chronic Hepatitis $\mathrm{B}$ and Drug-Induced Liver Disease. Clinics in Liver Disease, 18 (1): 165-78, 2013.

8- BADRUDDIN S.H., JAFRI S.M., AHMED A. and ABID S.: Dietary practices and beliefs of patients with chronic liver disease. The Journal of the Pakistan Medical Association JPMA, 49 (9): 216-20, 1999.

9- PURNAK T. and YILMAZ Y.: Liver disease and malnutrition. Best Practice \& Research Clinical Gastroenterology, 27: 619-29, 2013.

10- HANAFIAH K.H M., JUSTINA G.R. and ABRAHAM D.F.: Global epidemiology of hepatitis $C$ virus infection: New estimates of age-specific antibody to HCV seroprevalence. Hepat., 57 (4), 2013. 
11-National Nutrition Institute (NNI): www.nni.org.eg , 2017.

12- Abou Zeina, 2014.

13- MUIL D.: The Process Approach to ISO 9001, adding value by assessing existing business procedures. Retrieved at 4 April 2016, from www. qualitydigest.com/inside/ quality-insider-article/process-approach-iso-9001, 2014.

14- World Health Organization: Medical records manual: A guide for developing countries, WHO Regional Office for the Western Pacific. http://iris.wpro.who.int/handle/ 10665.1/10515 \& http://www.who.int/iris/handle/10665/ $208125,2006$.

15- SWEENEY J., BROOKS A. and LEAHY A.: Development of the Irish national patient perception of quality of care survey. International Journal for Quality in Health Care, 15: 163-8, 2003.

16- Department of Health and Human Services: Health Resources and Service Administration (U.S). Quality improvement, 2011.

17- MCKINLEY K. and ROBERTS C.: Patient satisfaction with out of hours primary medical care. Qual Health Care, 10 (1): 23-8, 2001.

18- Food and Nutrition Board: Institute of Medicine: Dietary Reference Intakes for Energy, Carbohydrate, Fiber, Fat, Fatty Acids, Cholesterol, Protein, and Amino Acids (Macronutrients). Washington, DC: National Academy Press, 2002.

19- JURAN J. and GODFREY A.: Juran's Quality Handbook (5 $5^{\text {th }}$ Edition). New York, USA: McGraw-Hill Professional Publishing, 1998.

20- BROWN J.: The Healthcare Quality Handbook: A Professional Resource and Study Guide (29 th ed., pp., CA $91104-$ 4926). USA, 2007.

21- ABOU-ZEINA H.A., ABDEL-HAI R., SOLIMAN M. and WAHBI G.H.: Health Services Utilization Pattern in Relation to Health Care Rights and Quality Perception: an Example From Rural Giza. The Egyptian Journal of Community Medicine, 25 (3), 2007.

22- HAMED N.: Utilization Pattern of Antenatal Care Services among a Group of Women in Fayoum Governorate, Public
Health and Community Medicine Department, Faculty of Medicine, Cairo University, 2014.

23- ABOU-ZEINA H.A.: Assessment of maternal and child health care delivered by three types of primary health care services. MD thesis, Public Health and Community Medicine Department, Faculty of Medicine, Cairo University, 1998.

24- MAC DONALD P., HATZEL T. and NEWMAN J.: Supervision, PRICOR service quality assessment series. Center for human services, Bethesda, 1992.

25- El-MOSALAMI M., SALEH Z., Al-MADAWI Y. and ElTOBGY A.: Utilization assessment of MCH services in Helwan. The Egypt. J. of Community Medicine, 11 (1), 1993.

26- SHRONTS E. and FISH J.: Hepatic failure. In Gottschlich MM, Matarese LE, Shronts EP (eds): Nutrition Support Dietetics: Core Curriculum, 2 nd ed. Silver Springs, MD: ASPEN, 311-26, 1993.

27- U.S. Department of Health and Human Services, Centers for Disease Control and Prevention, 1998.

28- REMIS S., HOGG R., KRAHN D., et al.: Estimating the number of blood transfusion recipients infected by Hepatitis C virus in Canada, 1960-85 and 1990-92. Health Canada, Ottawa, 1998.

29- Canadian Association for the Study of the Liver: The Management of Viral Hepatitis: Proceedings of the Canadian Consensus Conference on the Management of Viral Hepatitis. Can. J. Gastroenterol., 14 (Suppl B). CASL, Montreal, Quebec, 1999.

30- ZEMAN F.J.: Liver disease and alcoholism. In Clinical Nutrition and Dietetics, 2 nd ed., PP. 517-53. New York: Macmillan Publishing Company, 1991.

31- FATTOVITCH G., GIUSTINA G. and DEGOS F.: Morbidity and mortality in compensated cirrhosis type C: A retrospective follow-up study of 384 patients. Gastro, 112: 463-72, 1997.

32- National Information Centre for Health and Population in Egypt, 2010.

33- RDA, Food composition tables for Egypt National Nutrition Institute Cairo, 2nd edition, 2006. 


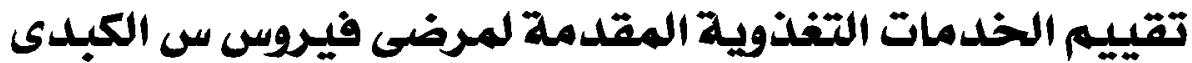

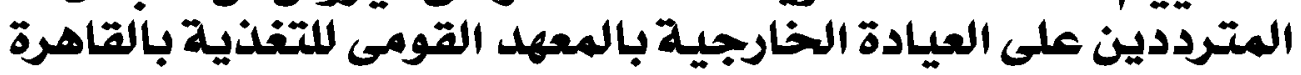

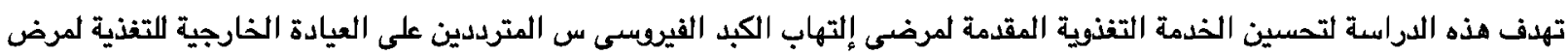

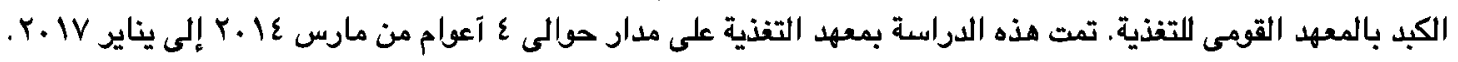

إستخدمت هذه الدراسة العديد من الآدوات لتقييم الخدمة التغنوية المقدمة لهزّلاء المرضي. تم تقديم إستبيان عن طريق المقابلة المباشرة

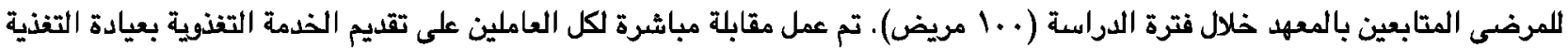
لمرضى الكبد بالمعهد.

إنتهت نتائج هذه الدراسة إلى وجود قصود فى الخدمة التفذوية المقدمة المرضى مع عدم وجود المعايير والقواعد الطبية منشودة آومونعة

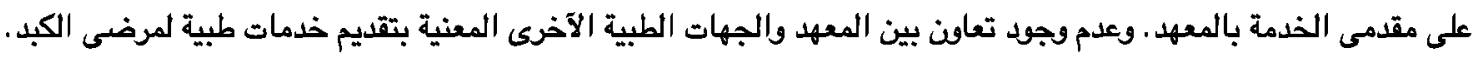

قدمت الدراسة إقتراحات بضرودة وجود وثيقة تعاون بين معهد التغذية ومعهد الكبد بخصوص علاج مرضى الكبدى س، آيضا تبنى ونشر المبر

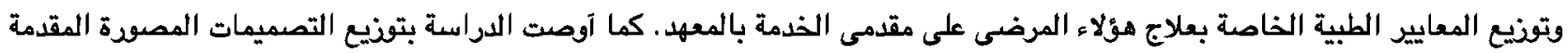

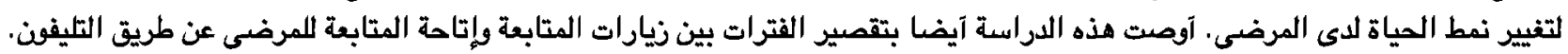

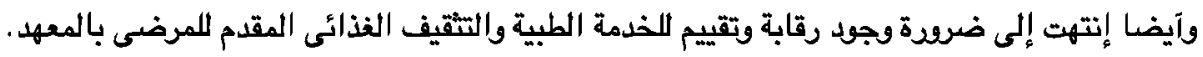

